\title{
High prevalence of gastroesophageal reflux symptoms in type 2 diabetics with hypoadiponectinemia and metabolic syndrome
}

\author{
Ayumu Hirata ${ }^{1}$, Ken Kishida ${ }^{1,2^{*}}$, Hideaki Nakatsuji', Kana Inoue ${ }^{1}$, Aki Hiuge-Shimizu', Tohru Funahashi ${ }^{1,2}$ and \\ lichiro Shimomura ${ }^{1}$
}

\begin{abstract}
Background: The prevalence of gastroesophageal reflux disease (GERD) has been increasing worldwide. Abdominal obesity or visceral fat accumulation rather than simple obesity is associated with GERD. Previous reports demonstrated the association between GERD and type 2 diabetes mellitus (T2DM). Signification of visceral fat accumulation and adiponectin in T2DM patients with GERD remains unclear. The present study investigated the relationships between GERD symptoms, visceral fat accumulation and adiponectin in subjects with T2DM.

Findings: The study (ADMIT study) subjects were 66 Japanese T2DM outpatients, who answered the questionnaire regarding GERD symptoms in Frequency Scale for the Symptoms of GERD (FSSG), and were measured visceral fat area by bioelectrical impedance analysis. Patients with FSSG scores of more than 8 were considered as positive. The prevalence of FSSG score $\geq 8$ and average FSSG score in T2DM subjects with the metabolic syndrome (Mets) were significantly higher compared to those without Mets. The prevalence of FSSG score $\geq 8$ and average FSSG score in T2DM subjects with low levels of serum adiponectin were significantly higher compared to those with high levels of serum adiponectin. Moreover, the combination of Mets and hypoadiponectinemia had a multiplicative effect on GERD symptom score $(p=0.047)$.
\end{abstract}

Conclusions: Our study showed that the coexistence of MetS and low levels of serum adiponectin was associated with the higher prevalence of FSSG score $\geq 8$ and the higher scores of GERD symptom in subjects with T2DM. Trial Registration

UMIN 000002271.

Keywords: gastroesophageal reflux symptom, metabolic syndrome, visceral fat, adiponectin

\section{Background}

The prevalence of gastroesophageal reflux disease (GERD) has been increasing worldwide [1], and is associated with impairment of quality of life (QOL), Barrett's esophagus, esophageal carcinogenesis and lifestylerelated diseases including sleep dysfunction, metabolic disorders and heart disease [2]. This increase is likely associated with the increased prevalence of obesity [2]. Obesity is shown to be an independent risk factor of GERD [3], through increased transient lower esophageal

\footnotetext{
* Correspondence: kkishida@imed2.med.osaka-u.ac.jp

'Department of Metabolic Medicine, Graduate School of Medicine, Osaka University, Suita, Osaka 565-0871, Japan

Full list of author information is available at the end of the article
}

sphincter relaxation, which is an important mechanism of GERD [4]. Abdominal obesity or visceral fat accumulation rather than simple obesity is associated with GERD [5,6]. With accumulation of visceral fat, circulating levels of adiponectin, a potential anti-inflammatory adipocytokine, are decreased, whereas circulating levels of proinflammatory cytokines, such as interleukin-6 (IL6), are increased. Low serum adiponectin levels were reported in subjects with esophago-gastro-duodenoscopic erosive esophagitis [7] or obese patients with GERD [8,9]. Visceral fat accumulation and dysregulated production of adipocytokines could exacerbate local inflammation at the esophagogastric junction. 
Table 1 Baseline characteristics of type 2 diabetic patients in the present study $(n=66)$

\begin{tabular}{|c|c|c|c|}
\hline & $\begin{array}{l}\text { Metabolic syndrome }(-) \\
(n=38)\end{array}$ & $\begin{array}{l}\text { Metabolic syndrome }(+) \\
(\mathrm{n}=28)\end{array}$ & $p$ value \\
\hline Gender, male/female & $18 / 20$ & $9 / 19$ & 0.210 \\
\hline Age, years & $63 \pm 1(36-78)$ & $67 \pm 2(40-88)$ & 0.101 \\
\hline Body mass index, $\mathrm{kg} / \mathrm{m}^{2}$ & $23.2 \pm 0.53(19.1-32.7)$ & $25.9 \pm 0.62(20.9-36.4)$ & 0.016 \\
\hline Waist circumference, $\mathrm{cm}$ & $84.1 \pm 1.3(69-104)$ & $91.4 \pm 1.3(65-109)$ & 0.002 \\
\hline Estimated visceral fat area, $\mathrm{cm}^{2}$ & $97 \pm 7(34-192)$ & $154 \pm 8(101-226)$ & $<0.0001$ \\
\hline Estimated glomerular filtration rate, $\mathrm{mL} / \mathrm{min}$ & $74.4 \pm 2.9(34.4-114)$ & $64.8 \pm 3.3(28.8-97.3)$ & 0.039 \\
\hline Blood glucose, mg/dL & $131 \pm 46(83-261)$ & $130 \pm 7(84-196)$ & 0.823 \\
\hline Fasting immunoreactive insulin, $\mu \mathrm{lU} / \mathrm{mL}(n=50)$ & $8.6 \pm 0.9(2.9-15.0)$ & $9.0 \pm 1.1(2.9-26.2)$ & 0.860 \\
\hline $\mathrm{HbA1c}$ (NGSP), \% & $7.1 \pm 0.1(5.9-10.6)$ & $7.2 \pm 0.2(5.6-9.6)$ & 0.702 \\
\hline Systolic blood pressure, $\mathrm{mmHg}$ & $130 \pm 2(105-180)$ & $134 \pm 3(110-160)$ & 0.708 \\
\hline Diastolic blood pressure, $\mathrm{mmHg}$ & $74 \pm 1(60-100)$ & $76 \pm 1(60-91)$ & 0.423 \\
\hline Triglyceride, mg/dL & $109 \pm 9(41-260)$ & $150 \pm 12(78-396)$ & 0.011 \\
\hline High-density lipoprotein cholesterol, mg/dL & $59 \pm 2(34-97)$ & $54 \pm 2(38-93)$ & 0.181 \\
\hline Low-density lipoprotein cholesterol, mg/dL & $111 \pm 5(57-208)$ & $116 \pm 6(57-203)$ & 0.550 \\
\hline Smoking (none/ex-/current-smoker) & 15/13/10 & $9 / 13 / 6$ & 0.604 \\
\hline Brinkman index & $531 \pm 106(0-3000)$ & $538 \pm 124(0-1800)$ & 0.622 \\
\hline Duration of diabetes, years & $10 \pm 1(1-38)$ & $11 \pm 1(1-38)$ & 0.529 \\
\hline Diabetic neuropathy & 7 & 10 & 0.104 \\
\hline Diabetic retinopathy (NDR/SDR/PDR) & $31 / 3 / 4$ & $18 / 6 / 4$ & 0.314 \\
\hline Diabetic nephropathy (stage I/II/III) & $25 / 8 / 5$ & $16 / 5 / 7$ & 0.359 \\
\hline Hypertension & 11 & 22 & 0.009 \\
\hline Dyslipidemia & 21 & 21 & 0.100 \\
\hline Medications for hypertension (CA/ACElorARB/ $\beta /$ diuretics $/ \alpha$ ) & $7 / 12 / 3 / 1 / 1$ & $15 / 18 / 3 / 2 / 1$ & 0.309 \\
\hline $\begin{array}{l}\text { Medications for diabetes } \\
\text { (SU/BG/aGl/glinide/Insulin) }\end{array}$ & $15 / 8 / 7 / 2 / 11$ & $11 / 11 / 8 / 0 / 5$ & 0.157 \\
\hline Medications for dyslipidemia (statins/fibrate) & $16 / 0$ & $16 / 0$ & 0.125 \\
\hline Serum adiponectin, $\mu \mathrm{g} / \mathrm{mL}$ (all; $\mathrm{n}=66$ ) & $9.8 \pm 0.9(2.3-33.5)$ & $8.4 \pm 1.1(2.2-21.5)$ & 0.235 \\
\hline adiponectin, $\mu \mathrm{g} / \mathrm{mL}$ (males) & $8.5 \pm 1.1(2.3-23.0)$ & $7.6 \pm 1.2(2.2-21.5)$ & 0.583 \\
\hline adiponectin, $\mu \mathrm{g} / \mathrm{mL}$ (females) & $11.2 \pm 1.5(3.9-33.5)$ & $9.8 \pm 1.9(4.2-11.5)$ & 0.576 \\
\hline Serum interleukin-6 (IL-6), pg/mL (all; n = 66) & $3.37 \pm 0.39(0.33-13.75)$ & $2.36 \pm 0.45(0.33-5.4)$ & 0.286 \\
\hline IL-6, pg/mL (males) & $3.92 \pm 0.59(0.81-13.75)$ & $2.16 \pm 0.63(0.33-5.4)$ & 0.075 \\
\hline IL-6, pg/mL (females) & $2.71 \pm 0.46(0.33-5.59)$ & $2.73 \pm 0.60(0.81-4.75)$ & 0.582 \\
\hline Serum TBARS, $\mathrm{nmol} / \mathrm{mL}$ (all, $\mathrm{n}=66$ ) & $4.49 \pm 0.15(2.68-7.30)$ & $4.68 \pm 0.18(3.01-6.56)$ & 0.423 \\
\hline TBARS, nmol/mL (males) & $4.45 \pm 0.20(2.68-6.10)$ & $4.44 \pm 0.21(3.01-6.56)$ & 0.958 \\
\hline TBARS, nmol/mL (females) & $4.53 \pm 0.24(3.24-7.30)$ & $5.23 \pm 0.35(4.00-6.20)$ & 0.112 \\
\hline FSSG scores & $2.5 \pm 1.4(0-24)$ & $8.6 \pm 1.8(0-24)$ & 0.015 \\
\hline$<8 / \geq 8$ & $33 / 5$ & 18/10 & 0.031 \\
\hline
\end{tabular}

mean \pm SEM or $n$ (range), NDR: non-diabetic retinopathy, SDR; simple diabetic retinopathy, PDR; proliferative diabetic retinopathy, SU; sulfonyl urea, BG; biguanide, $\alpha \mathrm{Gl}$; alpha glucosidase inhibitor, $C A$; calcium channel antagonist, ACEl; angiotensin converting enzyme inhibitor, ARB; angiotensin receptor blocker, $\beta$; $\beta$ blockade, $\alpha ; \alpha$ blockade, TBARS; thiobarbituric acid reactive substance. Estimated glomerular filtration rate $=194 \times$ creatinine $^{-1.094} \mathrm{x}$ age $\mathrm{e}^{-0.287}$. Significant level was set at $\mathrm{p}$ value $<0.05$ (bold type).

Previous reports demonstrated the association between GERD and type 2 diabetes mellitus (T2DM) in Japanese [10-12]. Signification of visceral fat accumulation and adiponectin in T2DM patients with GERD remains unclear. Ambulatory 24-h $\mathrm{pH}$ test-monitoring is the gold standard for diagnosing GERD [13]. This method, however, places pressure on patients. A questionnaire for the diagnosis of reflex disease (QUEST) was introduced and its usefulness for GERD diagnosis has been evaluated in Western countries [14]. However, QUEST have several problems that it was not easy to complete the questionnaire and evaluate therapeutic response of GERD in Japanese [15], and therefore, a new questionnaire for Japanese (FSSG; Frequency Scale for the Symptoms of GERD) was developed [16]. The present study investigated the relationships between GERD symptom score using FSSG, visceral fat accumulation and adiponectin in Japanese T2DM patients. 


\section{Methods and Procedures Study population}

Subjects were recruited from consecutive Japanese T2DM outpatients, who answered the questionnaire regarding GERD symptoms in FSSG, and were measured visceral fat area by bioelectrical impedance analysis (BIA) [17], in the "Diabetes \& Metabolic Station", Osaka University Hospital, between September 2009 and November 2011. Exclusion criteria included patients that were pregnant, a nursing mother, or a shift worker. Disease-related exclusion criteria included the following; malignant diseases, active peptic ulcer disease, or a past history of upper gastrointestinal surgery. Patients were also ineligible if they had received continuously acidsuppression drugs (histamine-2 blockers and protonpump inhibitors). The study subjects comprised 66 Japanese excluding subjects who were treated with pioglitazone $(\mathrm{n}=4)$, which is known to increase serum adiponectin levels in T2DM [18]. This study approved by the Medical Ethics Committee of Osaka University. All participants were Japanese and each gave a written informed consent. This study (ADMIT study) is registered under number UMIN 000002271.

https://upload.umin.ac.jp/cgi-open-bin/ctr/ctr.cgi?function $=$ brows $\&$ action $=$ brows $\&$ type $=$ summary $\&$ recptno $=$ R000002777\&language $=\mathrm{E}$

\section{GERD score}

The FSSG consisted of 12 questions, which were scored to indicate the frequency of symptoms as follows: never $=0$; occasionally $=1$; sometimes $=2$; often $=3$; and always $=4$. Patients with FSSG scores of more than 8 were considered as positive (When the cut-off score was set at 8 points, this test shows a sensitivity of $62 \%$, a specificity of $59 \%$, and an accuracy of $60 \%$ [16]). Based upon $80 \%$ power to detect statistically significant differences ( $p=0.05$; two-sided), a sample size of at least 25 patients in each group was required to demonstrate (total sample size $=50$ ), according to previous reports $[9,10]$.

\section{Anthropometry and laboratory measurements}

Venous blood samples were collected in the morning after overnight fast for measurements of each parameter and adiponectin (Otsuka Pharmaceutical Co., Tokushima, Japan, intra-coefficient of variation (CV); $<10 \%$, inter-CV < 10\%), IL-6 (Human IL-6 Quantikine ELISA Kit, R\&D Systems, USA, intra-CV; 7.3\%, inter-CV 7.7\%), as we previously reported $[18,19]$. Serum concentration of thiobarbituric acid-reacting substance (TBARS), an important biomarker of systemic oxidative stress reflecting serum lipid peroxidation products, was determined by the method of Yagi (Japan Institute for the Control of Aging, Nikken SEIL Co., Shizuoka, Japan), as reported previously by our group [18]. Diabetic retinopathy, nephropathy and peripheral neuropathy were diagnosed, as we previously reported [20]. The metabolic syndrome (Mets) was diagnosed according to the Japanese guidelines for Mets based on VFA $\geq 100 \mathrm{~cm}^{2}$ [21]. Briefly, the voltage recorded at the flank to the flow of current between the umbilicus and the back correlates significantly with visceral fat area (VFA) and is not influenced by subcutaneous fat. We reported previously that VFA estimated by BIA (eVFA) correlates significantly with that determined by computed tomography (CT) $(\mathrm{r}=$ $0.88, \mathrm{p}<0.0001)$ [17]. The CV of BIA with the value of $\mathrm{CT}$ was $0.89 \%$ in the standing position and late exhalation. A cutoff value for BIA-measured eVFA was 100 $\mathrm{cm}^{2}$ for both males and females as criteria to screen coming multiple obesity-related cardiovascular risk factors [22].

\section{Statistical analysis}

Data are mean \pm SEM, and compared by the $\chi^{2}$ and Mann-Whitney U-test in experiments of two groups. In all cases, a $p$ value $<0.05$ was considered statistically significant. All analyses were performed with the JMP Statistical Discovery Software 9.0 (SAS Institute, Cary, NC).

\section{Results}

Characteristics of subjects enrolled in the present study

The baseline characteristics of the patients are listed in Table 1 . The prevalence of FSSG score $\geq 8$ in subjects with T2DM was $23 \%(\mathrm{n}=15 / 66)$.

\section{Contribution of the metabolic syndrome and adiponectin on GERD symptoms}

To investigate the contribution of Mets on GERD symptoms, the subjects were divided into two groups, those without and with Mets. The prevalence of FSSG score $\geq$ 8 and average FSSG score in T2DM subjects with Mets were significantly higher compared to those without Mets (Figure 1A). Next, to investigate the contribution of adiponectin on GERD symptoms, the subjects were divided into two groups, those with low and high circulating levels of adiponectin (cutoff value $6.31 \mu \mathrm{g} / \mathrm{mL}$ for men, $8.62 \mu \mathrm{g} / \mathrm{mL}$ for women, median value, respectively). The prevalence of FSSG score $\geq 8$ and average FSSG score in T2DM subjects with low levels of serum adiponectin were significantly higher compared to those with high levels of serum adiponectin (Figure 1A). However, there was no significant difference of the prevalence of FSSG score $\geq 8$ and average FSSG score in between T2DM subjects with low and high levels of serum IL-6 (cutoff value $2.02 \mathrm{pg} / \mathrm{mL}$ for men, $2.65 \mathrm{pg} /$ $\mathrm{mL}$ for women, median value, respectively) (Figure 1A). 

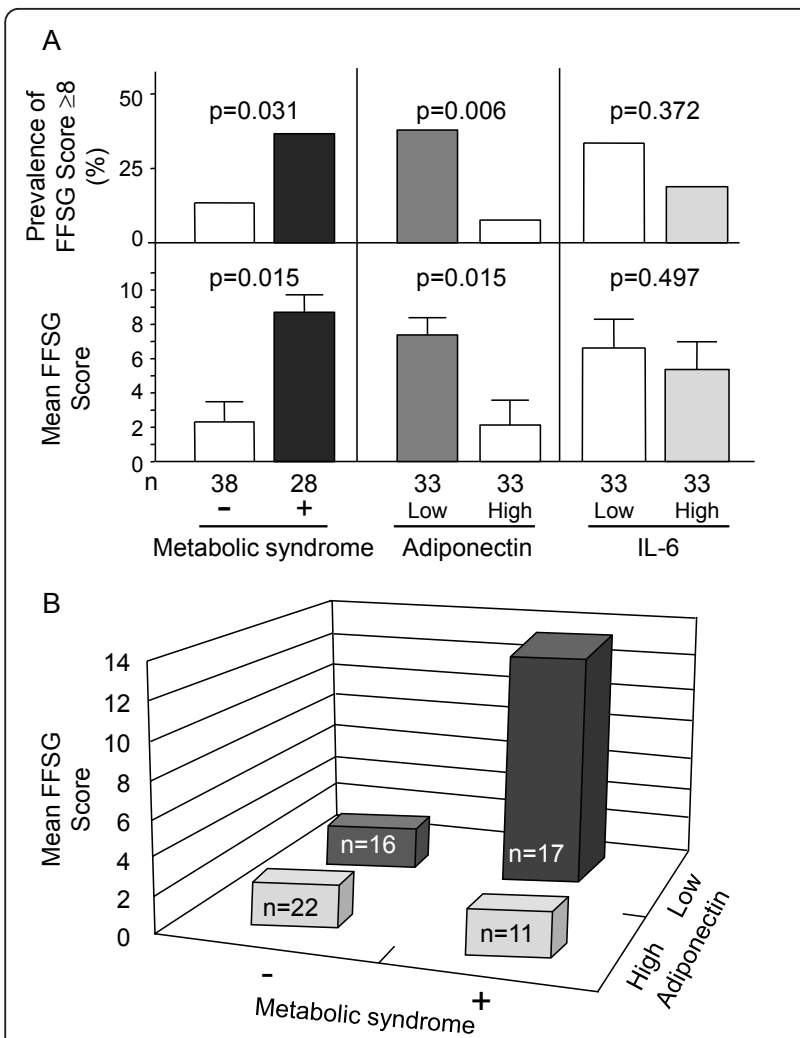

Figure 1

Figure $1 \mathrm{~A}$. Mean FSSG score in relation to the metabolic syndrome (Mets), serum adiponectin levels (serum adiponectin levels; cutoff value $6.31 \mu \mathrm{g} / \mathrm{mL}$ for men, $8.62 \mu \mathrm{g} / \mathrm{mL}$ for women, median value, respectively), and serum interleukin-6 (IL-6) (cutoff value $2.02 \mathrm{pg} / \mathrm{mL}$ for men, $2.65 \mathrm{pg} / \mathrm{mL}$ for women, median value, respectively). B. Comparison of prevalence of GERD symptoms (FSSG score $\geq 8$ ) and FSSG score between T2DM without and with Mets, and with low and high levels of serum adiponectin. Data are mean \pm SEM, and compared by the $\chi^{2}$ and Mann-Whitney $U$-test in experiments of two groups. All analyses were performed with the JMP Statistical Discovery Software 9.0 (SAS Institute, Cary, NC).

There was also no significant difference of the prevalence of FSSG score $\geq 8$ and average FSSG score in between T2DM subjects with low and high levels of serum TBARS (cutoff value $4.24 \mathrm{nmol} / \mathrm{mL}$ for men, 4.90 $\mathrm{nmol} / \mathrm{mL}$ for women, median value) (27\% versus $21 \%$; $=0.565,6.0 \pm 1.7$ versus $4.9 \pm 1.7 ; \mathrm{p}=0.648$, data no shown).

Finally, multiple regression analysis (adopted factors; age, sex, Mets, adiponectin, Mets $\times$ adiponectin) identified interaction term (Mets $\times$ adiponectin) as a significant determinant of GERD symptom score ( $p=0.047$, Figure $1 \mathrm{~B}$ ). These results indicated that the combination of Mets and hypoadiponectinemia had a multiplicative effect on GERD symptom score.

\section{Discussion}

The study of Japanese T2DM patients demonstrated for the first time that 1) the prevalence of T2DM with FSSG score $\geq 8$ was $23 \%(n=15 / 66), 2)$ the coexistence of Mets and low levels of serum adiponectin was associated with GERD symptoms. We have demonstrated that adiponectin suppresses inflammation in various organs, such as the heart, lung, aorta, kidney, liver, colon and pancreas [23]. Adiponectin may also play a protective role of erosive esophagitis. To clarify the protective effect of adiponectin on GERD, further experimental studies are required. On the other hand, increased systemic IL- 6 concentrations are associated with the pathophysiology of T2DM, with adipose tissue being the major source of this cytokine [24]. Exposure to components of the gastric refluxate is sufficient to stimulate esophageal cells to release a pro-inflammatory cytokine, IL-6, with the potential to mediate the esophageal motor abnormalities associated with GERD-induced esophagitis [25]. However, the present study show serum IL-6 levels are not associated with GERD symptoms in T2DM. Taken together, the results suggest that decreased anti-inflammatory cytokines rather than increased pro-inflammatory cytokines may be associated with the development of GERD-induced erosive esophagitis. We described here the prevalence and characteristics of Japanese diabetics with GERD symptoms, and it may be therefore necessary to diagnose a treatable GERD from the standpoint of prevention of lifestylerelated diseases as well as improvement of QOL in T2DM patients. Large-scale interventional trials, such as weight reduction, intensive anti-GERD and anti-diabetes (especially thiazolidinedione which is known to increase serum adiponectin) drugs or combinations of these therapies, should be provided to assess the effects of appropriate treatment on the outcome of T2DM patients with GERD symptoms.

Several limitations of this study must be considered. First, this is a cross-sectional study, making it difficult to establish a cause-effect relationship. Further prospective studies should be conducted in the future to analyze this relationship. Second, the results may not be applicable to females or non-Japanese populations. Third, the current study did not include the effects of alcohol intake, smoking habits, mental status, dietary habit and use of pharmacotherapy (such as nonsteroidal antiinflammatory drugs; NSAIDs). The current study found that the prevalence of FSSG score $\geq 8$ and average FSSG score were not influenced by smoking habits and use of NSAIDs. Finally, further replication studies of larger sample need to be designed including these confounding variables, such as potential factors to be influenced 
serum adiponectin levels, such as smoking status, use of pharmacotherapy (ACE-I/ARB, statin), in the future.

In conclusion, the coexistence of MetS and low levels of serum adiponectin was associated with the higher prevalence of GERD symptom in subjects with T2DM.

\section{List of Abbreviations}

BIA: bioelectrical impedance analysis; CV: coefficient of variation; eVFA: estimated visceral fat area; FSSG: Frequency Scale for the Symptoms of GERD; GERD: gastroesophageal reflux disease; IL-6: interleukin-6; Mets: metabolic syndrome; QOL: quality of life; TBARS: thiobarbituric acid-reacting substance; T2DM: type 2 diabetes mellitus.

\section{Acknowledgements and funding}

We thank all staff at "Diabetes \& Metabolic Station" for the excellent medical care, and Mrs. Miyuki Nakamura and Mr. Takeshi Nishimura for the excellent technical assistance. This research was supported in part by a Grant-in-Aid for Scientific Research on Innovative Areas (Research in a proposed research area) "Molecular Basis and Disorders of Control of Appetite and Fat Accumulation" (\#22126008, to T.F. and K.K.)

\section{Author details}

'Department of Metabolic Medicine, Graduate School of Medicine, Osaka University, Suita, Osaka 565-0871, Japan. ${ }^{2}$ Department of Metabolism and Atherosclerosis, Graduate School of Medicine, Osaka University, Suita, Osaka 565-0871, Japan.

\section{Authors' contributions}

A.H. and K.K. researched and analyzed data. K.K. also participated in the concept and design of the study, interpretation of data and reviewed/edited the manuscript. H.N., K.I. and A.H-S. recruited the patients and collected the data. T.F. and I.S. contributed to discussion and wrote the manuscript. All authors read and approved the final version of the manuscript.

\section{Competing interests}

K.K. and T.F. are members of the "Department of Metabolism and Atherosclerosis", a sponsored course endowed by Kowa Co. Ltd. and a company researcher is dispatched to the course. All other authors declare no competing interests.

Received: 21 December 2011 Accepted: 25 January 2012

Published: 25 January 2012

\section{References}

1. Sharma P, Wani S, Romero Y, Johnson D, Hamilton F: Racial and geographic issues in gastroesophageal reflux disease. Am J Gastroenterol 2008, 103:2669-80.

2. Roman S, Pandolfino JE: Environmental - lifestyle related factors. Best Pract Res Clin Gastroenterol 2010, 24:847-59.

3. Hampel H, Abraham NS, El-Serag HB: Meta-analysis: obesity and the risk for gastroesophageal reflux disease and its complications. Ann Intern Med 2005, 143:199-211.

4. Ayazi S, Hagen JA, Chan LS, DeMeester SR, Lin MW, Ayazi A, Leers JM, Oezcelik A, Banki F, Lipham JC, DeMeester TR, Crookes PF: Obesity and gastroesophageal reflux: quantifying the association between body mass index, esophageal acid exposure, and lower esophageal sphincter status in a large series of patients with reflux symptoms. J Gastrointest Surg 2009, 13:1440-47.

5. Lee HL, Eun CS, Lee OY, Jeon YC, Han DS, Yoon BC, Choi HS, Hahm JS, Ahn YH, Song SY: Association between erosive esophagitis and visceral fat accumulation quantified by abdominal CT scan. J Clin Gastroenterol 2009, 43:240-3.

6. Chung SJ, Kim D, Park MJ, Kim YS, Kim JS, Jung HC, Song IS: Metabolic syndrome and visceral obesity as risk factors for reflux oesophagitis: a cross-sectional case-control study of 7078 Koreans undergoing health check-ups. Gut 2008, 57:1360-5.

7. Kato M, Watabe K, Hamasaki T, Umeda M, Furubayashi A, Kinoshita K, Kishida O, Fujimoto T, Yamada A, Tsukamoto Y, Yamamoto S, Kamada Y,
Yoshida Y, Kiso S, Tsutsui S, Kihara S, Hayashi N, Matsuzawa Y: Association of low serum adiponectin levels with erosive esophagitis in men: an analysis of 2405 subjects undergoing physical check-ups. J Gastroenterol 2011, 46:1361-7.

8. Iwasaki E, Suzuki H, Sugino Y, lida T, Nishizawa T, Masaoka T, Hosoda H, Kangawa K, Hibi T: Decreased levels of adiponectin in obese patients with gastroesophageal reflux evaluated by videoesophagography: possible relationship between gastroesophageal reflux and metabolic syndrome. J Gastroenterol Hepatol 2008, 23:S216-21.

9. Moki F, Kusano M, Mizuide M, Shimoyama Y, Kawamura O, Takagi H, Imai T, Mori M: Association between reflux oesophagitis and features of the metabolic syndrome in Japan. Aliment Pharmacol Ther 2007, 26:1069-75.

10. Nishida T, Tsuji S, Tsujii M, Arimitsu S, Sato T, Haruna Y, Miyamoto T, Kanda T, Kawano S, Hori M: Gastroesophageal reflux disease related to diabetes: Analysis of 241 cases with type 2 diabetes mellitus. J Gastroenterol Hepatol 2004, 19:258-65.

11. Kase H, Hattori Y, Sato N, Banba N, Kasai K: Symptoms of gastroesophageal reflux in diabetes patients. Diabetes Res Clin Pract 2008, 79:e6-7.

12. Horikawa A, Ishii--Nozawa R, Ohguro M, Takagi S, Ohtuji M, Yamada M, Kuzuya N, Ujihara N, Ujihara M, Takeuchi K: Prevalence of GORD (gastrooesophageal reflux disease) in Type 2 diabetes and a comparison of clinical profiles between diabetic patients with and without GORD. Diabet Med 2009, 26:228-33.

13. Kahrilas PJ, Quigley EM: Clinical esophageal pH recording: a technical review for practice guideline development. Gastroenterology 1996, 110:1982-96.

14. Carlsson R, Dent J, Bolling-Sternevald E, Johnsson F, Junghard O, Lauritsen $\mathrm{K}$, Riley $\mathrm{S}$, Lundell $\mathrm{L}$ : The usefulness of a structured questionnaire in the assessment of symptomatic gastroesophageal reflux disease. Scand J Gastroenterol 1998, 33:1023-9.

15. Danjo A, Yamaguchi K, Fujimoto K, Saitoh $T$, Inamori M, Ando T, Shimatani T, Adachi K, Kinjo F, Kuribayashi S, Mitsufuji S, Fujiwara Y, Koyama S, Akiyama J, Takagi A, Manabe N, Miwa H, Shimoyama Y, Kusano M: Comparison of endoscopic findings with symptom assessment systems (FSSG and QUEST) for gastroesophageal reflux disease in Japanese centres. J Gastroenterol Hepatol 2009, 24:633-638

16. Kusano M, Shimoyama Y, Sugimoto S, Kawamura O, Maeda M, Minashi K, Kuribayashi S, Higuchi T, Zai H, Ino K, Horikoshi T, Sugiyama T, Toki M, Ohwada T, Mori M: Development and evaluation of FSSG: frequency scale for the symptoms of GERD. J Gastroenterol 2004, 39:888-91.

17. Ryo M, Maeda K, Onda T, Katashima M, Okumiya A, Nishida M, Yamaguchi T, Funahashi T, Matsuzawa Y, Nakamura T, Shimomura I: A new simple method for the measurement of visceral fat accumulation by bioelectrical impedance. Diabetes Care 2005, 28:451-3.

18. Nakatsuji H, Kishida K, Funahashi T, Shimomura I, Senri Study II Group: Three-month treatment with pioglitazone reduces circulating levels of thiobarbituric acid-reacting substances, a marker of reactive oxidative stress, without change in body mass index, in Japanese patients with type 2 diabetes. Atherosclerosis 2010, 212:243-5.

19. Kashine S, Kishida K, Funahashi T, Yasuda T, Okita K, Matsuzawa Y, Shimomura I: Selective contribution of waist circumference reduction on the improvement of sleep-disordered breathing in patients hospitalized with type 2 diabetes mellitus. Intern Med 2011, 50:1895-903.

20. Hirata A, Kishida K, Hiuge-Shimizu A, Nakatsuji H, Funahashi T, Shimomura I: Qualitative score of systemic arteriosclerosis by vascular ultrasonography as a predictor of coronary artery disease in type 2 diabetes. Atherosclerosis 2011, 219:623-9.

21. Teramoto T, Sasaki J, Ueshima H, Egusa G, Kinoshita M, Shimamoto K, Daida H, Biro S, Hirobe K, Funahashi T, Yokote K, Yokode M: Metabolic syndrome. J Atheroscler Thromb 2008, 15:1-5.

22. Okauchi Y, Kishida K, Funahashi T, Noguchi M, Ogawa T, Ryo M, Okita K, Iwahashi H, Imagawa A, Nakamura T, Matsuzawa Y, Shimomura I: Absolute value of bioelectrical impedance analysis-measured visceral fat area with obesity-related cardiovascular risk factors in Japanese workers. J Atheroscler Thromb 2010, 17:1237-45.

23. Ouchi N, Walsh K: Adiponectin as an anti-inflammatory factor. Clin Chim Acta 2007, 380:24-30

24. Spranger J, Kroke A, Möhlig M, Hoffmann K, Bergmann MM, Ristow M, Boeing H, Pfeiffer AF: Inflammatory cytokines and the risk to develop type 2 diabetes: results of the prospective population-based European 
Prospective Investigation into Cancer and Nutrition (EPIC)-Potsdam Study. Diabetes 2003, 52:812-7.

25. Rieder F, Cheng L, Harnett KM, Chak A, Cooper GS, Isenberg G, Ray M, Katz JA, Catanzaro A, O'Shea R, Post AB, Wong R, Sivak MV, McCormick T, Phillips M, West GA, Willis JE, Biancani P, Fiocchi C: Gastroesophageal reflux disease-associated esophagitis induces endogenous cytokine production leading to motor abnormalities. Gastroenterology 2007 132:154-65.

doi:10.1186/1743-7075-9-4

Cite this article as: Hirata et al: High prevalence of gastroesophageal reflux symptoms in type 2 diabetics with hypoadiponectinemia and metabolic syndrome. Nutrition \& Metabolism 2012 9:4.

Submit your next manuscript to BioMed Central and take full advantage of:

- Convenient online submission

- Thorough peer review

- No space constraints or color figure charges

- Immediate publication on acceptance

- Inclusion in PubMed, CAS, Scopus and Google Scholar

- Research which is freely available for redistribution

Submit your manuscript at www.biomedcentral.com/submit
C Biomed Central 